\title{
A SHARP BOUND FOR POSITIVE SOLUTIONS OF HOMOGENEOUS LINEAR DIOPHANTINE EQUATIONS
}

\author{
I. BOROSH
}

\begin{abstract}
Let $A$ be an $m \times n$ matrix of rank $r$ with integer entries. It is proved that if the system $A x=0$ has a nontrivial solution in nonnegative integers, then it has such a solution with entries bounded by the maximum of the absolute values of the $r \times r$ minors of $A$.
\end{abstract}

The following problem was considered in [1]: Given a system of linear equations with integer coefficients

$$
A x=B \text {, }
$$

and assuming that the system has a nontrivial solution in nonnegative integers, how small a bound $M=M(A, B)$ can be found such that the existence of such a solution to (1) with entries bounded by $M$ is guaranteed?

In the case of a single equation, a sharp bound $M$ was found in [1], namely the maximum of the absolute values of the coefficients. In the general case, it was conjectured that, if $r$ is the rank of $A, M$ can be chosen to be the maximum $M_{r}$ of the absolute value of the minors of order $r$ of the augmented matrix $(A, B)$. Such a bound would be, of course, sharp, but only weaker results were obtained in [1], even in the homogeneous case. It was proved [1, Theorem 3] that, in this case, $M$ can be chosen as the maximum of the absolute value of all the minors of $A$. The purpose of this note is to prove that the conjecture is true for the homogeneous case.

The existence of "small" solutions for homogeneous diophantine equations is used often in the theory of diophantine approximations, for example Siegel's Lemma ([4, p. 3] or [2, p. 106]). These result are not, however, directly comparable to the present result, since on the one hand they deal with integer solutions and are not concerned with the sign, and on the other hand the bounds are in terms of the coefficients and not in terms of the minors. We now state our result.

Received by the editors July 12, 1975 and, in revised form, March 19, 1976.

AMS (MOS) subject classifications (1970). Primary 15A06; Secondary 10B05.

1 This research was supported in part by the National Science Foundation under Grant No. GJ-43158. 
TheOREM. Let $A$ be an $m \times n$ matrix of rank $r$, with integer entries. Assume that the system

$$
A x=0,
$$

has a nontrivial nonnegative solution. Then it has a nontrivial solution in nonnegative integers not exceeding $M_{r}$, where $M_{r}$ is the maximum of the absolute value of all the minors of $A$ of order $r$.

Proof. We may assume without loss of generality that $m=r$. By Lemma 1 of [1], if $n-r \geqslant 2$ there exists an $m \times(n-1)$ submatrix $A^{\prime}$ of $A$ such that the system $A^{\prime} x=0$ has a nontrivial, nonnegative solution. Using this lemma repeatedly, we get finally an $m \times n^{*}$ submatrix $A^{*}$ of $A$, with rank $r^{*}=n^{*}$ -1 , and such that the system $A^{*} x=0$ has a nontrivial nonnegative solution. We may assume without loss of generality that $A^{*}$ is made out of the first $n^{*}$ columns of $A$ so that $A=\left(A^{*} \mid C\right)$.

There exists a unimodular $m \times m$ matrix $Q$ such that $Q A^{*}$ is in Hermite normal form (see e.g. [3, Chapter 3]).

$$
Q A^{*}=\left(\frac{H}{0}\right) \text { with } H=\left(\begin{array}{ccc}
h_{11} & \cdots & h_{1 n^{*}} \\
\bigcirc & \ddots & h_{r^{*} r^{*}} \\
h_{r^{*} n^{*}}
\end{array}\right)
$$

and the entries of $H$ are integers.

By Cramer's rule, the system $H x=0$ has a nontrivial nonnegative solution whose entries are minors of order $r^{*}$ of $H$. This solution is $x_{i}=H_{i}$, where $H_{i}$ is the determinant of the matrix obtained from $H$ by deleting the column $i$. It will, therefore, be enough to prove that every minor of order $r^{*}$ of $H$ is bounded by $M_{r}$. This is clear if $r^{*}=r$, we may therefore assume $r^{*}<r$, let

$$
Q A=A^{\prime}=\left(\frac{H}{0} \mid C^{\prime}\right)
$$

Let $H^{\prime}$ be an $r^{*} \times r^{*}$ submatrix of $H$ with $\operatorname{det} H^{\prime} \neq 0$. Since the rank of $A$ is $r$ we can find $r-r^{*}$ columns of $C^{\prime}$ such that if $D$ is the matrix formed by these columns then the square matrix

$$
G=\left(\begin{array}{c|c}
H^{\prime} \\
\hline 0
\end{array}\right)=\left(\begin{array}{l|l}
H^{\prime} & D_{1} \\
\hline 0 & D_{2}
\end{array}\right)
$$

is nonsingular. Since $Q$ is unimodular, $\operatorname{det} G$ is equal to some minor of $A$ of order $r$, therefore $|\operatorname{det} G| \leqslant M_{r}$. On the other hand, $\operatorname{det} G=\operatorname{det} H^{\prime} \cdot \operatorname{det} D_{2}$, and since $\operatorname{det} D_{2}$ is a nonzero integer, $|\operatorname{det} G| \geqslant \operatorname{det} H^{\prime} ;$ therefore $\left|\operatorname{det} H^{\prime}\right|$ $\leqslant M_{r}$. 


\section{REFERENCES}

1. I. Borosh and L. B. Treybig, Bounds on positive integer solutions of linear Diophantine equations, Proc. Amer. Math. Soc. 55 (1976), 299-304.

2. J. W. S. Cassels, An introduction to Diophantine approximation, Cambridge Tracts in Math. and Math. Phys., no. 45, Cambridge Univ. Press, New York, 1957. MR 19, 396.

3. Morris Newman, Integral matrices, Academic Press, New York and London, 1972. MR 49 \# 5038.

4. S. Lang, Introduction to transcendental numbers, Addison-Wesley, Reading, Mass., 1966. MR 35 \# 5397.

Department of Mathematics, Texas A \& M University, College Station, TeXas 77843 